\title{
Postmenopausal serum androgens, oestrogens and breast cancer risk: the European prospective investigation into cancer and nutrition
}

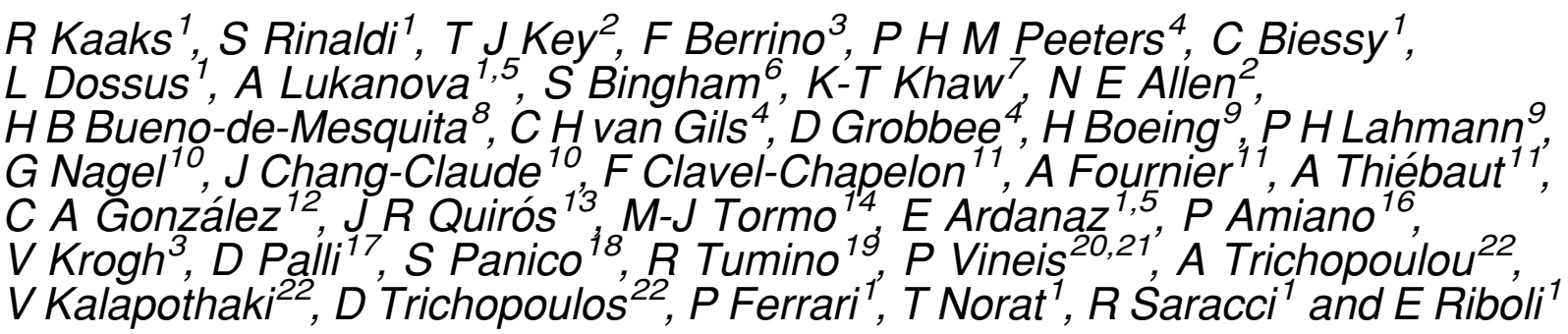

\footnotetext{
${ }^{1}$ International Agency for Research on Cancer (IARC-WHO), 150 cours Albert-Thomas, 69372 Lyon cedex 08, France

${ }^{2}$ Epidemiology Unit, Cancer Research UK, University of Oxford, Oxford, UK

${ }^{3}$ National Cancer Institute, Milan, Italy

${ }^{4}$ Julius Center for Health Sciences and Primary Care, University Medical Center, Utrecht, The Netherlands

${ }^{5} \mathrm{New}$ York University School of Medicine, New York, USA

${ }^{6}$ MRC Dunn Human Nutrition Unit, Welcome Trust/MRC Building, Cambridge, UK

${ }^{7}$ Clinical Gerontology Unit, Addenbrooke's Hospital, Cambridge, UK

${ }^{8}$ Centre for Nutrition and Health, National Institute for Public Health and the Environment, Bilthoven, The Netherlands

${ }^{9}$ Department of Epidemiology, German Institute of Human Nutrition, Potsdam-Rehbrücke, Germany

${ }^{10}$ Division of Clinical Epidemiology, German Cancer Research Center, Heidelberg, Germany

${ }^{11}$ INSERM, Institut Gustave Roussy, Villejuif, France

${ }^{12}$ Department of Epidemiology, Catalan Institute of Oncology, Barcelona, Spain

${ }^{13}$ Sección Información Sanitaria, Consejería de Salud y Servicios Sanitarios de Asturias, Asturias, Spain

${ }^{14}$ Epidemiology Department, Murcia Health Council, Murcia, Spain

${ }^{15}$ Public Health Institute of Navarre, Navarre, Spain

${ }^{16}$ Public Health Division of Gipuzkoa, Health Department of the Basque Country, Donostia-San Sebastian, Spain

${ }^{17}$ Molecular and Nutritional Epidemiology Unit, CSPO, Scientific Institute of Tuscany, Florence, Italy

${ }^{18}$ Department of Clinical and Experimental Medicine, Federico II University, Naples, Italy

${ }^{19}$ Cancer Registry, Azienda Ospedaliera 'Civile M.P. Arezzo', Ragusa, Italy

${ }^{20}$ University of Turin, Turin, Italy

${ }^{21}$ Imperial College of Science, Technology and Medicine, London, UK

${ }^{22}$ University of Athens Medical School, Athens, Greece
}

(Requests for offprints should be addressed to R Kaaks; Email: kaaks@iarc.fr)

\begin{abstract}
Considerable experimental and epidemiological evidence suggests that elevated endogenous sex steroids - notably androgens and oestrogens - promote breast tumour development. In spite of this evidence, postmenopausal androgen replacement therapy with dehydroepiandrosterone (DHEA) or testosterone has been advocated for the prevention of osteoporosis and improved sexual wellbeing. We have conducted a case-control study nested within the European Prospective Investigation into Cancer and Nutrition. Levels of DHEA sulphate (DHEAS), ( $\triangle 4$-androstenedione), testosterone, oestrone, oestradiol and sex-hormone binding globulin (SHBG) were measured in prediagnostic serum samples of 677 postmenopausal women who subsequently developed breast cancer and 1309 matched control subjects. Levels of free testosterone and free oestradiol were calculated from absolute concentrations of testosterone, oestradiol and SHBG. Logistic regression models were used to estimate relative risks of breast cancer by quintiles of hormone concentrations. For all sex steroids the androgens as well as the oestrogens - elevated serum levels were positively associated with breast cancer risk, while SHBG levels were inversely related to risk. For the androgens, relative risk
\end{abstract}


estimates (95\% confidence intervals) between the top and bottom quintiles of the exposure distribution were: DHEAS 1.69 (1.23-2.33), androstenedione 1.94 (1.40-2.69), testosterone 1.85(1.33-2.57) and free testosterone 2.50 (1.76-3.55). For the oestrogens, relative risk estimates were: oestrone 2.07 (1.42-3.02), oestradiol 2.28 (1.61-3.23) and free oestradiol (odds ratios 2.13 (1.52-2.98)). Adjustments for body mass index or other potential confounding factors did not substantially alter any of these relative risk estimates. Our results have shown that, among postmenopausal women, not only elevated serum oestrogens but also serum androgens are associated with increased breast cancer risk. Since DHEAS and androstenedione are largely of adrenal origin in postmenopausal women, our results indicated that elevated adrenal androgen synthesis is a risk factor for breast cancer. The results from this study caution against the use of DHEA(S), or other androgens, for postmenopausal androgen replacement therapy.

Endocrine-Related Cancer (2005) 12 1071-1082

\section{Introduction}

Most of the established epidemiological risk factors for breast cancer are related to alterations in endogenous hormone metabolism (Pike et al. 1993, Russo \& Russo 1999, Russo et al. 2000, Key et al. 2001). A young age at first full-term pregnancy, high parity and prolonged duration of breast feeding all independently protect against tumour development, and collectively have been estimated to explain up to about $50 \%$ of the excess risk of breast cancer in industrially developed societies compared with developing countries (Collaborative Group on Hormonal Factors in Breast Cancer 2002). These protective effects have been postulated to be due to the effects of pregnancyassociated alterations in endogenous hormone metabolism on mammary epithelial differentiation (Russo et al. 2000) and/or proliferation (Pike et al. 1993, Sivaraman \& Medina 2002), or on the numbers of mammary stem cells, which could be the susceptible target cells from which breast tumours develop (Baik et al. 2004). By contrast, early menarche, late menopause and use of postmenopausal hormone replacement therapy (HRT) are all associated with increased risk of breast cancer, and these increases in risk are generally interpreted as being the result of a longer lifetime exposure to elevated sex steroids, particularly oestrogens, which may inhibit apoptosis and stimulate proliferation of the mammary duct epithelium (Pike et al. 1993, Key et al. 2001). Finally, among postmenopausal women, excess weight increases breast cancer risk, and this too is generally believed to be due to adiposity-related increases in total and bioavailable endogenous oestrogens (Bianchini et al. 2002, Lahmann et al. 2004).

Besides oestrogens, elevated endogenous androgens have also long been implicated as a potential risk factors for breast cancer (Grattarola 1973, Grattarola et al. 1974, Secreto et al. 1991). Normal and malignant mammary epithelial cells also have androgen receptors (Dimitrakakis et al. 2002, Liao \& Dickson 2002), indicating specific responsiveness to androgens, and epidemiological studies have generally indicated positive relationships of breast cancer risk with postmenopausal plasma androgen levels. Paradoxically, however, premenopausal plasma levels of dehydroepiandrosterone (DHEA) or its sulphate (DHEAS) have been found to be inversely related to breast cancer risk (Adams 1998). In experimental studies (for reviews see Dimitrakakis et al. 2002, Liao et al. 2002) bioactive androgens (testosterone, dihydrotestosterone) or their precursors (e.g. DHEA or DHEAS) have been found to either antagonize or enhance breast cell proliferation or mammary tumour growth (Dimitrakakis et al. 2002, Liao \& Dickson 2002). The inverse associations of breast cancer risk with premenopausal DHEA levels and the apparent protective effects of androgens in some of the experimental studies have been cited to support the use of postmenopausal androgen replacement therapy for the prevention of osteoporosis, improved wellbeing and/or sexual functioning and, possibly, breast cancer prevention (Spark 2002, Labrie et al. 2003).

Results from at least 10 prospective cohort studies on breast cancer risk in relation to prediagnostic blood levels of sex steroids among postmenopausal women have been published (Wysowski et al. 1987, BarrettConnor et al. 1990, Gordon et al. 1990, Garland et al. 1992, Helzlsouer et al. 1994, Toniolo et al. 1995, Berrino et al. 1996, Dorgan et al. 1996, 1997, Thomas et al. 1997a, Zeleniuch-Jacquotte et al. 1997, Hankinson et al. 1998, Cauley et al. 1999, Kabuto et al. 2000). However, most of these studies were based on rather small numbers of cases, were conducted in diverse populations in the USA, Europe and Japan, and used heterogeneous study protocols, blood collection procedures and laboratory methods for hormone assays. A combined re-analysis of the pooled data 
from nine of these studies - including up to a maximum of 663 incident cases of breast cancer and 1102 control subjects, depending on the specific hormonal parameters examined - showed approximately twofold increases in breast cancer risk between women in the upper versus the lower quintiles of all of the sex steroids examined: DHEA or DHEAS, $(\Delta 4-$ androstenedione), testosterone, oestrone, total oestradiol and indices of bioavailable oestradiol not bound to sex hormone-binding globulin (SHBG) (Key et al. 2002). We present results from a case-control study nested within the European Prospective Investigation into Cancer and Nutrition (EPIC) - a multi-centre prospective study aimed at investigating the relationships between nutrition and other lifestyle factors, metabolism, genetic predisposition and cancer risk. In total, our study included 677 incident cases of breast cancer and 1309 matched control subjects, and thus was equal in size to all previously published cohort studies combined, but from an entirely European population in which standardized methods were used for collection of blood samples and questionnaire data, and for hormone assays.

\section{Study subjects and methods}

The EPIC cohort consisted of about 370000 women and 150000 men, recruited between 1992 and 1998 in 23 research centres spread over ten western European countries (Riboli et al. 2002). All subjects provided extensive standardized questionnaire data on diet and non-dietary variables, as well as anthropometric measurements. About 240000 women and 140000 men also provided a blood sample. All data on exposure and follow-up for cancer incidence and vital status from the different EPIC centres were coded in a standardized manner, checked for coding errors and internal consistency of subjects' questionnaire information, and entered into an extensively documented ORACLE database housed centrally at the International Agency for Research on Cancer (IARC, Lyon, France). All subjects provided written consent for their participation in the EPIC cohort study and for the use of questionnaire data and blood samples for research purposes. The present study included breast cancer cases and control subjects from 17 recruitment centres in seven of the participating countries: France, The Netherlands, the UK, Germany, Spain, Italy and Greece. Norway was not included in the present study because blood samples have been collected only recently on a subsample of cohort participants and so far only very few cases of breast cancer have been accumulated after blood collection; Denmark and
Sweden were not included because independent studies on breast cancer risk and endogenous sex hormones have been, or are being, conducted separately.

\section{Questionnaire data and anthropometry}

Questionnaire data on non-dietary lifestyle and health factors included menstrual and reproductive history, current and previous use of oral contraceptives (OC) and postmenopausal HRT, history of previous illness and disorders or surgical operations, lifetime history of tobacco smoking and consumption of alcoholic beverages, physical activity, level of education and socioeconomic status, and brief occupational history. Fully standardized questionnaires were used in all seven countries contributing to the present study. In all countries included in the present analysis, except part of the cohort recruited through the Oxford research centre, height, weight, and waist and hip circumferences were measured according to standardized protocols, in light dress. In part of the Oxford cohort, height, weight and body circumferences were self-reported. All measurements were reported to the nearest $\mathrm{cm}$ (height and body circumferences) and to the nearest $\mathrm{kg}$ (weight).

\section{Collection and storage of blood samples}

Blood samples were collected according to a standardized protocol. From each subject, $30 \mathrm{ml}$ blood was drawn using $10 \mathrm{ml}$ Safety Monovettes (Sartstedt, Nümbrecht, Germany). Filled syringes were kept at $5-10^{\circ} \mathrm{C}$, protected from light and transferred to a local laboratory for further processing and aliquoting. Two of the three syringes contained trisodium citrate as anticoagulant for the preparation of blood plasma, buffy coat and red cells, and one dry syringe was used to prepare serum. After centrifugation $(1550 \mathrm{~g}$ for $20 \mathrm{~min}$ ), blood fractions (serum, plasma, red cells and buffy coat) were aliquoted in 28 plastic straws of $0.5 \mathrm{ml}$ each (12 plasma, 8 serum, 4 erythrocytes and 4 buffy coat for DNA), which were heat-sealed and stored under liquid nitrogen $\left(-196^{\circ} \mathrm{C}\right)$. Half of the 28 aliquots were stored locally and the other half centrally at IARC.

\section{Determination of menopausal status at blood donation}

Women were considered menopausal when they reported not having had any menses over the past 12 months or when they reported bilateral ovariectomy. When questionnaire data were missing or incomplete or when women reported previous hysterectomy, women were considered postmenopausal when they 
were older than 55 years. All other women, for whom data were equivocal, were excluded from the present study. Women who at the time of blood sampling were using hormone replacement therapy (HRT), a factor that can mask the menopausal transition, were also excluded (see criteria for case-control selection below).

\section{Follow-up for cancer incidence and vital status}

In The Netherlands, the UK, Spain and Italy incident cancer cases were identified through record linkage with regional cancer registries. In Germany, France and Greece follow-up was based on a combination of methods, including health insurance records, cancer and pathology registries, and active follow-up through study subjects and their next-of-kin. Data on vital status in most EPIC study centres were collected from mortality registries at the regional or national level, in combination with data collected by active follow-up (Greece). For each EPIC study centre, closure dates of the study period were defined as the latest dates of complete follow-up for both cancer incidence and vital status (dates varied between centres).

\section{Selection of case and control subjects}

Case and control subjects were selected among women who were postmenopausal and not using any HRT at the time of blood donation and who had no previous diagnosis of cancer (except non-melanoma skin cancer). Case subjects were women who developed breast cancer after their recruitment into the EPIC study and blood donation, and before the end of the study period, for each study centre defined by the latest end-date of complete follow-up. Out of 677 incident cases of breast cancer, 63 had a carcinoma in situ and all others had an invasive tumour.

For each case subject, up to two control subjects were chosen at random among appropriate risk sets consisting of all cohort members alive and free of cancer (except non-melanoma skin cancer) at the time of diagnosis of the index case. An incidence density sampling protocol for control selection was used, such that controls could include subjects who became a case later in time, while each control could also be sampled more than once. Matching criteria was the study centre where the subjects were enrolled in the cohort, age at enrolment ( \pm 6 months), follow-up time since blood donation $( \pm 3$ months $)$ and time of the day of blood collection $( \pm 1 \mathrm{~h})$. Cases and controls were also matched on time between blood withdrawal and last consumption of food or drink $(<3 \mathrm{~h}, 3-6 \mathrm{~h},>6 \mathrm{~h})$, in view of further studies in which breast cancer risk will be related to endogenous insulin levels.

\section{Hormone assays}

Testosterone and DHEAS concentrations in serum were measured by radioimmunoassays (Immunotech, Marseilles, France). Androstenedione, oestradiol and oestrone concentrations were measured by a radioimmunoassay with a double-antibody system for the separation of free and bound antigen (Diagnostic Systems Laboratories Inc., Webster, TX, USA). Assays were performed in batches of 76 serum samples, analysed together on the same day and with the same immunoassay kit. Serum and samples of case and control subjects that were matched together were systematically analysed within the same batch (i.e. with a single immunoassay kit). SHBG was measured by a solid phase 'sandwich' immunoradiometric assay (Cis-Bio International, Gif-sur-Yvette, France). Mean intra-batch coefficients of variation were $7.0 \%$ for DHEAS, $10.8 \%$ for testosterone, $4.8 \%$ for androstenedione, $10.2 \%$ for oestrone, $5.8 \%$ for oestradiol and $8.0 \%$ for SHBG. All hormone assays were performed by the laboratory of the Hormones and Cancer Group, at IARC. The laboratory personnel performing the hormonal assays were blinded as to the case-control status of the study subjects. DHEA was not measured, because usually its levels show very high correlations $(>0.9)$ with DHEAS. Serum concentrations of free testosterone and free oestradiol, unbound to SHBG or albumin, were calculated from the absolute concentrations of each of the steroids and SHBG using mass action equations, and assuming a constant serum albumin concentration of $43 \mathrm{~g} / 1$ (Rinaldi et al. 2002).

\section{Statistical analyses}

In all analyses, levels of SHBG and sex steroids were log-transformed to normalize their distributions. An analysis of variance, adjusting for age, case-control status and laboratory batch, was used to examine the study centre as a determinant of measured hormone levels. Correlations between the hormones and anthropometric indices, adjusting for age and laboratory batch, were calculated from the sums of squares in generalized linear regression models. Statistical significance of case-control differences in mean hormonal exposure levels were evaluated by paired comparisons ( $t$-tests) of case values versus the average of the two matched controls in each case-control set (Rosner 1982). For binary variables, significance of case-control differences was tested by Chi-square test. Relative risks (odds ratios; OR) for disease at different 
Table 1 Baseline characteristics of case and control subjects, all study centres combined (means [ $5^{\text {th }}-95^{\text {th }}$ percentile ranges]; population percentages)

\begin{tabular}{lccc}
\hline & Cases & Controls & P for difference \\
\hline Number of subjects & 677 & 1309 & \\
Age at recruitment & $60.4[51.4-70.1]$ & $60.3[51.2-70.1]$ & 0.46 \\
Age at blood donation & $60.6[51.4-70.4]$ & $60.6[51.5-70.4]$ & 0.13 \\
Years since menopause (recruitment) & $11.0[2.3-23.3]$ & $11.4[2.3-24.3]$ & -21 \\
Years since menopause (diagnosis) & $13.6[4.0-26.0]$ & - & - \\
Age at diagnosis & $63.0[54.0-74.0]$ & - & - \\
Years between blood collection and diagnosis & $2.9[0.1-6.3]$ & $16.3 \%$ & 0.29 \\
Previous use of hormones for menopause & $14.5 \%$ & $34.7 \%$ & 0.05 \\
Previous use of oral contraceptive & $30.1 \%$ & $85.6 \%$ & 0.46 \\
Ever had full-term pregnancy & $86.3 \%$ & $25.6[20.0-33.0]$ & 0.02 \\
Age at first full-term pregnancy & $26.0[20.0-35.0]$ & $13.3[11.0-16.0]$ & 0.12 \\
Age at menarche & $13.2[11.0-16.0]$ & $26.8[20.6-35.6]$ & 0.05 \\
Body-mass index & $27.2[21.1-36.0]$ & $0.81[0.72-0.92]$ & 0.84 \\
Waist-hip ratio & $0.81[0.71-0.92]$ & & \\
\hline
\end{tabular}

serum hormone levels were calculated by conditional logistic regression models, where the serum levels of the various hormones and binding proteins were examined both as continuous variables and by quintiles. The quintiles were based on the logtransformed variable distributions of the control subjects from all EPIC centres combined. Likelihood ratio tests were used to assess linear trends in ORs with increasing exposure level as a continuous variable, or with assigned quantitative scores 1, 2, 3, 4 and 5 for the quintile categories. Confidence intervals (C.I.; 95\%) were computed using the standard errors of the pertinent regression coefficients. The effects of additional potential confounders (other than the matching criteria, controlled for by design) were examined by including additional regression terms into the logistic regression models. Potential confounders included anthropometry (as continuous variables), previous use of exogenous hormones (OCs, HRT; as binary variables 'use/never used'), age at first full-term pregnancy (years) (five categories), number of full-term pregnancies (five categories), age at menarche (years) (five categories) and age at menopause (years) (six categories). Chi-square tests were used to examine heterogeneity of association of breast cancer risk with hormone levels - e.g. by study centre, body mass index (BMI) categories, previous use of HRT.

\section{Results}

Hormone measurements were completed for a total of 677 cases of breast cancer and 1309 cancer-free control subjects, with small percentages of missing values for each of the hormones. On average, women were 60.6 years old at the time of blood donation, with a 5-95th percentile range of 51.5-70.4 years (Table 1). At the time of recruitment, the average time since menopause was around 11 years, for both breast cancer cases and control subjects. Cancer diagnosis occurred on average 13.6 years after menopause, with a $5-95$ th percentile range of 4.0-26.0 years, and 2.9 years after blood donation (5-95th percentile range 0.1-6.3 years). Compared with control subjects, cancer patients were significantly older at first full-term pregnancy, had a higher BMI and less frequently reported past use of OCs, but showed no significant differences in previous use of HRT, age at menarche or mean waist-hip ratio (Table 1).

The prevalence of major breast cancer risk factors varied between the 17 EPIC recruitment centres involved in this study. For control subjects, the average BMI varied between $24.1 \mathrm{~kg} / \mathrm{m}^{2}$ in the French cohort to over $29.0 \mathrm{~kg} / \mathrm{m}^{2}$ in Greece and all five Spanish centres. The prevalence of reported previous HRT use (i.e. before blood donation) also varied widely between the study centres, from $0 \%$ in Ragusa (southern Italy), Asturias and Murcia (Spain) to 39\% in Heidelberg (Germany). The latter variations could not be explained by differences in age or years since menopause, but rather reflected cultural differences in medication practices, especially between northern and southern Europe.

Levels of all sex steroids and SHBG varied significantly between the 17 study centres: after adjusting for age and case-control status, the per cent variance in hormone concentrations explained by study centre was $4.6 \%$ for DHEAS, $5.6 \%$ for androstenedione, $8.0 \%$ for SHBG, $10.9 \%$ for testosterone, 
Table 2 Pearson correlation coefficients on log transformed data between hormonal variables adjusted for age, case-control status and laboratory batch

\begin{tabular}{|c|c|c|c|c|c|c|c|c|c|}
\hline & $\begin{array}{c}\text { Free } \\
\text { Testosterone }\end{array}$ & $\Delta 4$ & DHEAS & SHBG & Oestrone & Oestradiol & $\begin{array}{c}\text { Free } \\
\text { Oestradiol }\end{array}$ & BMI & Waist \\
\hline Testosterone & $0.90^{*}$ & $0.66^{*}$ & $0.69^{*}$ & $-0.06 \sim$ & $0.46^{*}$ & $0.49^{*}$ & $0.46^{*}$ & $0.06 \#$ & $0.07 \sim$ \\
\hline Free Testosterone & & $0.62^{*}$ & $0.65^{\star}$ & $-0.47^{\star}$ & $0.48^{*}$ & $0.50^{*}$ & $0.61^{*}$ & $0.23^{\star}$ & $0.25^{\star}$ \\
\hline$\triangle 4$ & & & $0.60^{*}$ & $-0.09 \sim$ & $0.41^{*}$ & $0.44^{*}$ & $0.43^{*}$ & 0.01 & 0.01 \\
\hline DHEAS & & & & $-0.13^{\star}$ & $0.47^{*}$ & $0.45^{\star}$ & $0.45^{\star}$ & 0.03 & $0.05 \#$ \\
\hline SHBG & & & & & $-0.17^{\star}$ & $-0.17^{*}$ & $-0.43^{*}$ & $-0.37^{\star}$ & $-0.40^{\star}$ \\
\hline Oestrone & & & & & & $0.69^{\star}$ & $0.68^{*}$ & $0.22^{*}$ & $0.23^{*}$ \\
\hline Oestradiol & & & & & & & $0.95^{\star}$ & $0.23^{*}$ & $0.24^{*}$ \\
\hline Free Oestradiol & & & & & & & & $0.33^{*}$ & $0.35^{\star}$ \\
\hline
\end{tabular}

$\# p<0.05, \sim p<0.01,{ }^{*} p<0.0001$ (two-sided tests).

Table 3 Geometric mean [95\% confidence intervals] of steroid hormones by case-control status

\begin{tabular}{lccc}
\hline & Cases & Controls & $P$ for difference \\
\hline Testosterone $(\mathrm{nmol} / \mathrm{l})$ & $1.27[1.22-1.33]$ & $1.15[1.11-1.18]$ & $<0.0001$ \\
Free testosterone $(\mathrm{pmol} / \mathrm{l})$ & $21.6[20.5-22.8]$ & $18.8[18.1-19.5]$ & $<0.0001$ \\
Androstenedione $(\mathrm{nmol} / \mathrm{l})$ & $3.31[3.16-3.47]$ & $2.99[2.89-3.09]$ & 0.0003 \\
DHEAS $(\mu \mathrm{mol} / \mathrm{l})$ & $2.15[2.04-2.26]$ & $1.93[1.86-2.00]$ & 0.0002 \\
SHBG $(\mathrm{nmol} / \mathrm{l})$ & $31.5[30.1-33.0]$ & $33.4[32.3-34.5]$ & 0.04 \\
Oestrone $(\mathrm{pmol} / \mathrm{l})$ & $157.9[152.4-163.6]$ & $144.9[143.3-148.6]$ & $<0.0001$ \\
Oestradiol $(\mathrm{pmol} / \mathrm{l})$ & $99.1[95.5-102.8]$ & $89.7[87.4-92.1]$ & $<0.0001$ \\
Free Oestradiol $(\mathrm{pmol} / \mathrm{l})$ & $2.64[2.53-2.74]$ & $2.34[2.28-2.41]$ & $<0.0001$ \\
\hline
\end{tabular}

$12.3 \%$ for oestradiol and $17.7 \%$ for oestrone. These percentages reduced to a range of 0.6 for testosterone to 1.9 for oestradiol, after further adjustment for the laboratory batch in which the samples were analysed. However, since subjects from a given study centre were, as much as possible, analysed within the same analytical batch, these variance decomposition models did not allow a clear distinction of variation in true hormone levels between centres from the variation attributable to between-batch, analytical errors. A separate analysis, of values obtained for standard control samples that had been included in all batches, indicated that the variance of analytical between-batch errors was very small in comparison with the overall population variance, with values ranging from $0.1 \%$ for DHEAS to $1.5 \%$ for androstenedione.

Adjusting for laboratory batch, age and casecontrol status, serum concentrations of the androgens (DHEA, androstenedione, testosterone) were all directly correlated amongst each other, as were the two oestrogens, and calculated values of free testosterone and free oestradiol, unbound to SHBG, correlated strongly with the absolute concentrations of each hormone. All three androgens also showed moderate positive correlations with both oestrone and oestradiol. BMI showed mild inverse correlations with SHBG, and direct correlations with oestrone, oestradiol, free oestradiol and free testosterone (Table 2).

For all study centres combined, geometric mean levels of all sex steroids were significantly higher and SHBG levels significantly lower among cases compared with control subjects (Table 3 ). In conditional logistic regression analyses, these differences were reflected in statistically significant increases in breast cancer risk with increasing absolute concentrations (quintile levels) of androgens (DHEAS, androstenedione, testosterone) and oestrogens (oestrone, oestradiol), as well as with increasing free testosterone and free oestradiol concentrations (Fig. 1). The strongest relative risks were for free testosterone and oestradiol, with ORs of $2.50 \quad(95 \% \quad$ C.I. $=1.76-3.55)$ and $2.28(1.61-3.23)$ respectively for top versus bottom quintiles.

When centre-specific quintile cut points were used, relative risk estimates were very close to those from analyses with EPIC-wide cut points. Furthermore, the estimated relationships of breast cancer risk with hormone levels, either expressed as tertile levels or on a continuous scale, showed no statistically significant heterogeneity between study centres. 


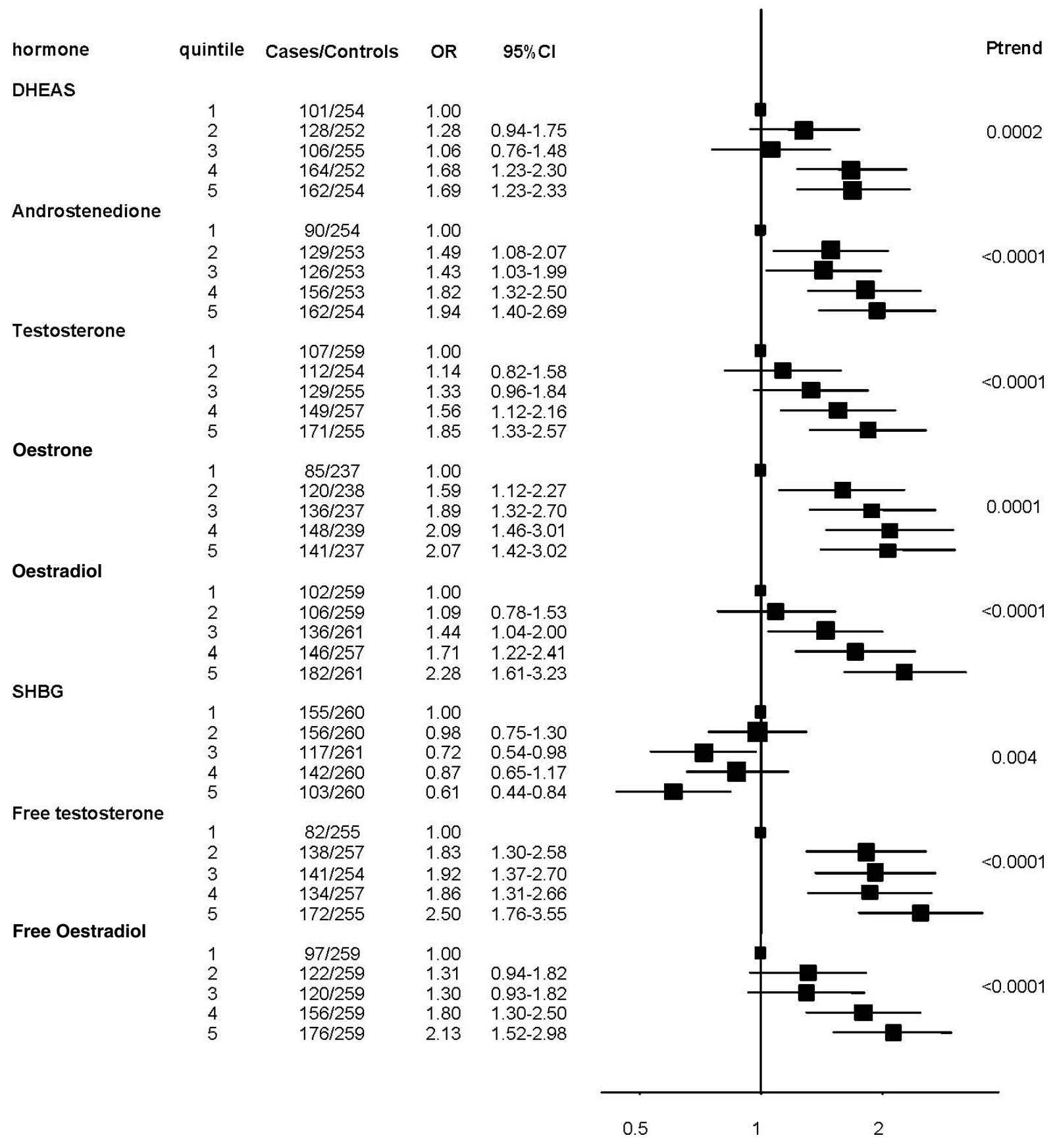

Figure 1 Relative risk of breast cancer among postmenopausal women by quintiles of serum steroid concentrations. OR estimated by conditional logistic regression with study centre, age at blood donation, time of day of blood donation and fasting status at blood donation as matching factors for breast cancer cases and control subjects.

Relative risk estimates remained virtually unchanged after adjustments for BMI, age at first full-term pregnancy, age at menarche, number of full-term pregnancies and previous use of OCs or postmenopausal HRT (data not shown). Furthermore, relative risk estimates showed no significant heterogeneity between cases that had a cancer diagnosis within 2 years of blood donation and cases with a later diagnosis (results not shown). Relative risks also did not differ significantly between women who did or did 
not report previous use of HRT, contrary to the findings in some previous studies (Hankinson et al. 1998, Key et al. 2002). Finally, relative risks remained virtually unaltered when the analysis was restricted to invasive breast tumours. For the subset of carcinomas in situ $(n=63)$ a statistical subgroup analysis indicated that the association of each of the hormonal parameters (as continuous variables) with risk was in the same direction as that for the invasive tumours (results not shown).

Using multivariate models, it was examined whether risk remained related to levels of total or free oestrogens independently of androgen levels and vice versa (data not shown). In all models, using hormone levels on a continuous $(\log 2)$ scale, relative risk estimates for oestrogens were mildly attenuated after adjustment for androgen levels; likewise, relative risks with respect to androgens were also attenuated after adjustment for oestrogen levels. Generally, the androgen-adjusted relative risks for oestrogen levels were stronger, and more strongly significant, than oestrogen-adjusted relative risks for levels of the androgens. These results were very similar to those of the pooled re-analysis of previous prospective cohort studies (Key et al. 2002).

\section{Discussion}

This large, multicentric cohort study showed a roughly twofold increase in breast cancer risk among postmenopausal women who had elevated blood concentrations of total and bioavailable androgens and oestrogens. Relative risk estimates remained virtually unaltered after adjustment for potential confounders, or restriction of the statistical analysis to subgroups, and were strikingly similar to those from the pooled re-analysis of nine previous prospective cohort studies (Key et al. 2002), which altogether included roughly the same numbers of cases and controls as our study. Our results are also in line with those from prospective studies relating breast cancer risk to urinary levels of androgens and oestrogens (Key et al. 1996, Thomas et al. 1997b, Onland-Moret et al. 2003).

Compared with traditional case-control studies, the prospective design of our study avoided inverse causation bias that might be caused by alterations in endogenous hormone levels due to metabolic effects of a (large) tumour, or to anti-tumour treatments, psychological stress and lifestyle changes after cancer diagnosis. Furthermore, the prospective design ensured the selection of control subjects from the same, welldescribed study base in which the breast cancer cases occurred, and allowed a careful matching of case and control subjects according to baseline characteristics.
Our study had a high degree of standardization of recruitment and blood collection protocols, questionnaire data and hormone measurements, across subpopulations with heterogeneous lifestyles and cancer risks. Although the average hormone levels varied between the study centres, this variation represented only a small proportion of the overall between-subject variation, and relative risk estimates were very similar when quintile levels were based on EPIC-wide cut points or on centre-specific cut points. The consistency of our study results across the EPIC recruitment centres, in spite of considerable heterogeneity of the population subgroups in terms of previous HRT use, average BMI, parity, mean age at menarche and other risk factors for breast cancer, suggests that our findings can be generalized to many other populations in affluent societies. Although associations varied in magnitude between the 17 study centres included in this analysis, this variation appeared to have been due mostly to random sampling differences. Generalizability is further corroborated by the great similarity of relative risk estimates between our study and the pooled re-analysis of previous prospective studies, which were also conducted in quite diverse populations of Europe, the USA and Japan.

The direct immunoassays used in the present project were previously validated by comparison with indirect assays after organic extraction and chromatographic prepurification, and showed very high correlations $(r>0.80)$ for total concentrations of both androgens and oestrogens (Rinaldi et al. 2001). Calculated values of free testosterone and oestradiol were also found to have high correlations with reference measurements based on equilibrium dialysis (Rinaldi et al. 2002). In addition, measurements of sex steroids in a single blood sample are known to reflect women's long-term levels after menopause quite accurately. Previous studies have shown reasonably high intra-class correlations between measured concentrations of DHEAS, testosterone and androstenedione, as well as of oestrogens, in blood samples taken over time periods of 1 year and longer (values around 0.8-0.9 for DHEAS and total testosterone, around 0.9 for SHBG and around 0.6-0.7 for androstenedione and oestradiol (Cauley et al. 1991, Hankinson et al. 1995, Muti et al. 1996, Lukanova et al. 2004)). Nevertheless, variations over time in true hormone levels and laboratory measurement errors will have almost certainly led to some attenuation of our relative risk estimates.

A vast body of epidemiological and experimental evidence indicates a central role of oestrogens in promoting breast tumour development (Russo \& Russo 1999, Russo et al. 2000, Key et al. 2001). The 
strongest direct epidemiological evidence, perhaps, comes from observations of increased breast cancer risk among postmenopausal women using oestrogen or combined oestrogen-plus-progestin replacement therapy (Collaborative Group on Hormonal Factors in Breast Cancer 1997, Beral 2003, Chlebowski et al. 2003, Kenemans \& Bosman 2003), even though a recent randomized intervention study did not show any excess in breast cancer incidence among women given replacement of oestrogens alone (Anderson et al. 2004).

Our observation of an increased risk of breast cancer among the women with higher serum oestrogen concentrations is in line with this large body of evidence implicating oestrogens in mammary tumour development. It has been debated, however, whether circulating levels of bioavailable oestradiol and oestrone are themselves the key determinant of oestrogen levels and bioactivity in the mammary tissue, or whether they merely reflect the magnitude of extraglandular oestrogen synthesis in breast and other peripheral tissues from androgenic precursors from the adrenal glands and ovaries. All enzymes necessary for the transformation of androgenic precursors into oestrone and oestradiol are present in normal mammary tissues and breast tumour specimens (Liao et al. 2002, Labrie et al. 2003), and it has been determined that the concentration of oestradiol present in breast tumours of postmenopausal women is 10- to 20 -fold greater than that present in plasma (Pasqualini et al. 1996, Blankenstein et al. 1999). Oestrogenic activity in mammary tissue may thus be largely determined by levels of local ('intracrine') synthesis of oestradiol from oestrone and androgenic precursors.

Regarding DHEAS, androstenedione and testosterone, our study, like previous prospective studies (Wang et al. 2000, Key et al. 2002), has shown that in postmenopausal women each of these androgens is directly associated with breast cancer risk. One likely explanation for these direct associations is that elevated DHEA(S), androstenedione, and to some extent perhaps bioavailable testosterone, may lead to increased mammary and adipose tissue synthesis of oestrogens, which in turn may enhance tumour development. The direct correlations between serum androgens and oestrogens support the concept that androgen levels may be a co-determinant of amounts of oestrogens formed peripherally.

In postmenopausal women, plasma levels of DHEAS and androstenedione are mostly determined by adrenal and/or ovarian synthesis and secretion, with comparatively minor contributions from extraglandular conversions. More than $90 \%$ of circulating
DHEAS, and after menopause about $80 \%$ of circulating androstenedione, originates directly from the adrenal glands and this, plus the direct association of breast cancer risk with serum DHEAS and androstenedione concentrations, indicates that elevated adrenal androgen synthesis is a risk factor for breast cancer. The relative contribution of ovarian androgen synthesis to increased circulating levels and breast cancer risk is more difficult to assess. Although the ovaries are the predominant glandular source of circulating testosterone, only about $40 \%$ of circulating testosterone comes from ovaries, and most of the remainder is formed by extraglandular conversion of androgenic precursors (Longcope 1986, Adashi 1994). Although bilateral ovariectomy has been found to mildly reduce circulating androgen levels, especially androstenedione and testosterone, in postmenopausal women (Sluijmer et al. 1995, Kenemans \& Bosman 2003), ovariectomy after the age of $45-50$ years does not appear to decrease breast cancer risk (Schairer et al. 1997, Kreiger et al. 1999).

There is strong evidence that postmenopausal HRT use - oestrogens alone or oestrogens combined with progestins - leads to a moderate increase in breast cancer risk (Collaborative Group on Hormonal Factors in Breast Cancer 1997, Beral 2003, Chlebowski et al. 2003, Kenemans \& Bosman 2003). As a possible alternative to this type of postmenopausal hormone replacement, DHEA and/or DHEAS have been proposed as a form of physiological HRT at menopause to compensate for age-related decreases in serum levels and, as an important precursor for synthesis of more active androgens in peripheral 'intracrine' tissues. According to the proponents of this concept, DHEA (or DHEAS) would have the advantage over other androgens that at physiological doses it is converted into more active androgens (testosterone, dihydrotestosterone) and/or oestrogens only in those specific target tissues that possess the appropriate enzymes for such conversion, thus limiting the action of the sex steroids to those same tissues (Labrie et al. 2003). DHEA(S) would also have the advantage of increasing bone mineral density as well as improving well-being and libido, without significant effects, for example, on endometrial tissue (Spark 2002, Labrie et al. 2003). Although theoretically these potential advantages may seem attractive, our observation of an association of DHEAS levels with breast cancer risk strongly caution against the use of DHEA(S) for postmenopausal hormone replacement.

From a prevention perspective, an important question is how the excess of endogenous sex steroids that predisposes to increased breast cancer risk may be 
reduced. Our data showed associations of BMI with oestrone, total and free oestradiol and free testosterone, but no clear relationship with DHEAS, androstenedione or total testosterone. Adjusting for BMI did not alter relative risk estimates of breast cancer for different levels of androgens or oestrogens. Conversely, adjusting for total serum androgens also did not alter relative risks for BMI much, whereas adjustments for total or free oestrogens did substantially reduce the BMI-risk relationship (data not shown). Observations were very similar in the pooled re-analysis of previous prospective cohort studies (Key et al. 2002) and, taken together, these various observations suggest that weight reduction may reduce peripheral synthesis and circulating levels of oestrogens but not of total androgens.

In conclusion, our large prospective cohort study has confirmed earlier evidence that among postmenopausal women breast cancer risk is directly related to circulating levels of both androgens and oestrogens. We favour the interpretation that, at the mammary tissue level, breast tumour development may be driven more by excess oestrogen than androgens. However, besides excess weight (adiposity), which leads to increased peripheral conversion of androgenic precursors into oestrogens, elevated adrenal and possibly ovarian androgen production appears to contribute independently to increased breast cancer risk by providing additional substrate for conversion into oestrogens. While weight control may contribute to breast cancer prevention (Bianchini et al. 2002), most likely through reduced oestrogen synthesis, further studies are needed to establish more clearly the physiologic origins especially of the relative androgen excess in women at increased risk of breast cancer, and to understand which changes in lifestyle or other interventions may be used to lower circulating androgen levels. Finally, our data provide a strong indication against the use of androgens for postmenopausal hormone replacement.

\section{Acknowledgements}

The EPIC study was funded by 'Europe Against Cancer' Programme of the European Commission (SANCO), Ligue contre le Cancer (France), Société 3M (France), Mutuelle Générale de l'Education Nationale, Institut National de la Santé et de la Recherche Médicale (INSERM), German Cancer Aid, German Cancer Research Center, German Federal Ministry of Education and Research, Danish Cancer Society, Health Research Fund (FIS) of the Spanish Ministry of Health (RCEP-03/09), the participating regional governments and institutions of Spain, Cancer Research UK, Medical Research Council (UK), Stroke Association (UK), British Heart Foundation, Department of Health (UK), Food Standards Agency (UK), Wellcome Trust (UK), Greek Ministry of Health, Greek Ministry of Education, Italian Association for Research on Cancer, Italian National Research Council, Dutch Ministry of Public Health, Welfare and Sports, Dutch Ministry of Health, Dutch Prevention Funds, LK Research Funds, Dutch Zorg Onderzoek Nederland (ZON), World Cancer Research Fund (WCRF), Swedish Cancer Society, Swedish Scientific Council, Regional Government of Skane (Sweden) and Norwegian Cancer Society. Specific study results of this case-control study nested within EPIC, presented in this paper, were obtained with financial support from the National Cancer Institute (USA) (grant no. 1U01CA98216-01)

The authors declare that there is no conflict of interest that would prejudice the impartiality of this scientific work.

\section{References}

Adams JB 1998 Adrenal androgens and human breast cancer: a new appraisal. Breast Cancer Research and Treatment 51 183-188.

Adashi EY 1994 The climacteric ovary as a functional gonadotropin-driven androgen-producing gland. Fertility and Sterility 62 20-27.

Anderson GL, Limacher M, Assaf AR, Bassford T, Beresford SA, Black H, Bonds D, Brunner R, Brzyski R, Caan B et al. 2004 Effects of conjugated equine estrogen in postmenopausal women with hysterectomy: the Women's Health Initiative randomized controlled trial. Journal of the American Medical Association 291 1701-1712.

Baik I, Becker PS, DeVito WJ, Lagiou P, Ballen K, Quesenberry PJ \& Hsieh CC 2004 Stem cells and prenatal origin of breast cancer. Cancer Causes and Control 15 517-530.

Barrett-Connor E, Friedlander NJ \& Khaw KT 1990 Dehydroepiandrosterone sulfate and breast cancer risk. Cancer Research 50 6571-6574.

Beral V 2003 Breast cancer and hormone-replacement therapy in the Million Women Study. Lancet $362419-427$.

Berrino F, Muti P, Micheli A, Bolelli G, Krogh V, Sciajno R, Pisani P, Panico S \& Secreto G 1996 Serum sex hormone levels after menopause and subsequent breast cancer. Journal of the National Cancer Institute 88 291-296.

Bianchini F, Kaaks R \& Vainio H 2002 Overweight, obesity, and cancer risk. Lancet Oncology 3 565-574.

Blankenstein MA, van dJ, Maitimu-Smeele I, Donker GH, de Jong PC, Daroszewski J, Szymczak J, Milewicz A 
\& Thijssen JH 1999 Intratumoral levels of estrogens in breast cancer. Journal of Steroid Biochemistry and Molecular Biology 69 293-297.

Cauley JA, Gutai JP, Kuller LH \& Powell JG 1991 Reliability and interrelations among serum sex hormones in postmenopausal women. American Journal of Epidemiology 133 50-57.

Cauley JA, Lucas FL, Kuller LH, Stone K, Browner W \& Cummings SR 1999 Elevated serum estradiol and testosterone concentrations are associated with a high risk for breast cancer. Study of Osteoporotic Fractures Research Group. Annals of Internal Medicine 130 270-277.

Chlebowski RT, Hendrix SL, Langer RD, Stefanick ML, Gass M, Lane D, Rodabough RJ, Gilligan MA, Cyr MG, Thomson CA et al. 2003 Influence of estrogen plus progestin on breast cancer and mammography in healthy postmenopausal women: the Women's Health Initiative Randomized Trial. Journal of the American Medical Association 289 3243-3253.

Collaborative Group on Hormonal Factors in Breast Cancer 1997 Breast cancer and hormone replacement therapy: collaborative reanalysis of data from 51 epidemiological studies of 52,705 women with breast cancer and 108,411 women without breast cancer. Collaborative Group on Hormonal Factors in Breast Cancer. Lancet 350 1047-1059.

Collaborative Group on Hormonal Factors in Breast Cancer 2002 Breast cancer and breastfeeding: collaborative reanalysis of individual data from 47 epidemiological studies in 30 countries, including 50,302 women with breast cancer and 96,973 women without the disease. Lancet 360 187-195.

Dimitrakakis C, Zhou J \& Bondy CA 2002 Androgens and mammary growth and neoplasia. Fertility and Sterility 77 (Suppl 4) S26-S33.

Dorgan JF, Longcope C, Stephenson HE Jr, Falk RT, Miller R, Franz C, Kahle L, Campbell WS, Tangrea JA \& Schatzkin A 1996 Relation of prediagnostic serum estrogen and androgen levels to breast cancer risk. Cancer Epidemiology, Biomarkers and Prevention 5 533-539.

Dorgan JF, Stanczyk FZ, Longcope C, Stephenson HE Jr, Chang L, Miller R, Franz C, Falk RT \& Kahle L 1997 Relationship of serum dehydroepiandrosterone (DHEA), DHEA sulfate, and 5-androstene-3 beta, 17 beta-diol to risk of breast cancer in postmenopausal women. Cancer Epidemiology, Biomarkers and Prevention 6 177-181.

Garland CF, Friedlander NJ, Barrett-Connor E \& Khaw KT 1992 Sex hormones and postmenopausal breast cancer: a prospective study in an adult community. American Journal of Epidemiology 135 1220-1230.

Gordon GB, Bush TL, Helzlsouer KJ, Miller SR \& Comstock GW 1990 Relationship of serum levels of dehydroepiandrosterone and dehydroepiandrosterone sulfate to the risk of developing postmenopausal breast cancer. Cancer Research 50 3859-3862.
Grattarola R 1973 Androgens in breast cancer. I. Atypical endometrial hyperplasia and breast cancer in married premenopausal women. American Journal of Obstetrics and Gynecology 116 423-428.

Grattarola R, Secreto G, Recchione C \& Castellini W 1974 Androgens in breast cancer. II. Endometrial adenocarcinoma and breast cancer in married postmenopausal women. American Journal of Obstetrics and Gynecology 118 173-178.

Hankinson SE, Manson JE, Spiegelman D, Willett WC, Longcope C \& Speizer FE 1995 Reproducibility of plasma hormone levels in postmenopausal women over a 2-3 year period. Cancer Epidemiology, Biomarkers and Prevention 4 649-654.

Hankinson SE, Willett WC, Manson JE, Colditz GA, Hunter DJ, Spiegelman D, Barbieri RL \& Speizer FE 1998 Plasma sex steroid hormone levels and risk of breast cancer in postmenopausal women. Journal of the National Cancer Institute 90 1292-1299.

Helzlsouer KJ, Alberg AJ, Bush TL, Longcope C, Gordon GB \& Comstock GW 1994 A prospective study of endogenous hormones and breast cancer. Cancer Detection and Prevention 18 79-85.

Kabuto M, Akiba S, Stevens RG, Neriishi K \& Land CE 2000 A prospective study of estradiol and breast cancer in Japanese women. Cancer Epidemiology, Biomarkers and Prevention 9 575-579.

Kenemans P \& Bosman A 2003 Breast cancer and post-menopausal hormone therapy. Best Practice and Research. Clinical Endocrinology and Metabolism 17 123-137.

Key TJ, Wang DY, Brown JB, Hermon C, Allen DS, Moore JW, Bulbrook RD, Fentiman IS \& Pike MC 1996 A prospective study of urinary oestrogen excretion and breast cancer risk. British Journal of Cancer 73 1615-1619.

Key TJ, Verkasalo PK \& Banks E 2001 Epidemiology of breast cancer. Lancet Oncology 2 133-140.

Key T, Appleby P, Reeves G \& Endogenous Hormones and Breast Cancer Collaborative Group 2002 Endogenous sex hormones and breast cancer in postmenopausal women: reanalysis of nine prospective studies. Journal of the National Cancer Institute 94 606-616.

Kreiger N, Sloan M, Cotterchio M \& Kirsh V 1999 The risk of breast cancer following reproductive surgery. European Journal of Cancer 35 97-101.

Labrie F, Luu-The V, Labrie C, Belanger A, Simard J, Lin SX \& Pelletier G 2003 Endocrine and intracrine sources of androgens in women: inhibition of breast cancer and other roles of androgens and their precursor dehydroepiandrosterone. Endocrine Reviews 24 152-182.

Lahmann PH, Hoffmann K, Allen N, van Gils CH, Khaw KT, Tehard B, Berrino F, Tjonneland A, Bigaard J, Olsen A et al. 2004 Body size and breast cancer risk: findings from the European Prospective Investigation into Cancer and Nutrition (EPIC). International Journal of Cancer 111 762-771. 
Liao DJ \& Dickson RB 2002 Roles of androgens in the development, growth, and carcinogenesis of the mammary gland. Journal of Steroid Biochemistry and Molecular Biology 80 175-189.

Longcope C 1986 Adrenal and gonadal androgen secretion in normal females. Clinics in Endocrinology and Metabolism 15 213-228.

Lukanova A, Lundin E, Micheli A, Arslan A, Ferrari P, Rinaldi S, Krogh V, Lenner P, Shore RE, Biessy C et al. 2004 Circulating levels of sex steroid hormones and risk of endometrial cancer in postmenopausal women. International Journal of Cancer 108 425-432.

Muti P, Trevisan M, Micheli A, Krogh V, Bolelli G, Sciajno R \& Berrino F 1996 Reliability of serum hormones in premenopausal and postmenopausal women over a one-year period. Cancer Epidemiology, Biomarkers and Prevention 5 917-922.

Onland-Moret NC, Kaaks R, van Noord PA, Rinaldi S, Key T, Grobbee DE \& Peeters PH 2003 Urinary endogenous sex hormone levels and the risk of postmenopausal breast cancer. British Journal of Cancer 88 1394-1399.

Pasqualini JR, Chetrite G, Blacker C, Feinstein MC, Delalonde L, Talbi M \& Maloche C 1996 Concentrations of estrone, estradiol, and estrone sulfate and evaluation of sulfatase and aromatase activities in pre- and postmenopausal breast cancer patients. Journal of Clinical Endocrinology and Metabolism 81 1460-1464.

Pike MC, Spicer DV, Dahmoush L \& Press MF 1993 Estrogens, progestogens, normal breast cell proliferation, and breast cancer risk. Epidemiologic Reviews 15 17-35.

Riboli E, Hunt KJ, Slimani N, Ferrari P, Norat T, Fahey M, Charrondiere UR, Hemon B, Casagrande C, Vignat J et al. 2002 European Prospective Investigation into Cancer and Nutrition (EPIC): study populations and data collection. Public Health Nutrition 5 1113-1124.

Rinaldi S, Dechaud H, Biessy C, Morin-Raverot V, Toniolo P, Zeleniuch-Jacquotte A, Akhmedkhanov A, Shore RE, Secreto G, Ciampi A et al. 2001 Reliability and validity of commercially available, direct radioimmunoassays for measurement of blood androgens and estrogens in postmenopausal women. Cancer Epidemiology, Biomarkers and Prevention 10 757-765.

Rinaldi S, Geay A, Dechaud H, Biessy C, ZeleniuchJacquotte A, Akhmedkhanov A, Shore RE, Riboli E, Toniolo P \& Kaaks R 2002 Validity of free testosterone and free estradiol determinations in serum samples from postmenopausal women by theoretical calculations. Cancer Epidemiology, Biomarkers and Prevention 11 1065-1071.

Rosner B 1982 A generalization of the paired t-test. Applied Statistics 31 9-13.
Russo J \& Russo IH 1999 Cellular basis of breast cancer susceptibility. Lancet Oncology 11 169-178.

Russo J, Hu YF, Yang X \& Russo IH 2000 Developmental, cellular, and molecular basis of human breast cancer. Journal of the National Cancer Institute Monographs 17-37.

Schairer C, Persson I, Falkeborn M, Naessen T, Troisi R \& Brinton LA 1997 Breast cancer risk associated with gynecologic surgery and indications for such surgery. International Journal of Cancer 70 150-154.

Secreto G, Toniolo P, Berrino F, Recchione C, Cavalleri A, Pisani P, Totis A, Fariselli G \& Di Pietro S 1991 Serum and urinary androgens and risk of breast cancer in postmenopausal women. Cancer Research 51 2572-2576.

Sivaraman L \& Medina D 2002 Hormone-induced protection against breast cancer. Journal of Mammary Gland Biology and Neoplasia 7 77-92.

Sluijmer AV, Heineman MJ, De Jong FH \& Evers JL 1995 Endocrine activity of the postmenopausal ovary: the effects of pituitary down-regulation and oophorectomy. Journal of Clinical Endocrinology and Metabolism 80 2163-2167.

Spark RF 2002 Dehydroepiandrosterone: a springboard hormone for female sexuality. Fertility and Sterility 77 (Suppl 4) S19-S25.

Thomas HV, Key TJ, Allen DS, Moore JW, Dowsett M, Fentiman IS \& Wang DY $1997 a$ A prospective study of endogenous serum hormone concentrations and breast cancer risk in post-menopausal women on the island of Guernsey. British Journal of Cancer 76 401-405.

Thomas HV, Reeves GK \& Key TJ 1997b Endogenous estrogen and postmenopausal breast cancer: a quantitative review. Cancer Causes and Control 8 922-928.

Toniolo PG, Levitz M, Zeleniuch-Jacquotte A, Banerjee S, Koenig KL, Shore RE, Strax P \& Pasternack BS 1995 A prospective study of endogenous estrogens and breast cancer in postmenopausal women. Journal of the National Cancer Institute 87 190-197.

Wang DY, Allen DS, De Stavola BL, Fentiman IS, Brussen J, Bulbrook RD, Thomas BS, Hayward JL \& Reed MJ 2000 Urinary androgens and breast cancer risk: results from a long-term prospective study based in Guernsey. British Journal of Cancer 82 1577-1584.

Wysowski DK, Comstock GW, Helsing KJ \& Lau HL 1987 Sex hormone levels in serum in relation to the development of breast cancer. American Journal of Epidemiology 125 791-799.

Zeleniuch-Jacquotte A, Bruning PF, Bonfrer JM, Koenig KL, Shore RE, Kim MY, Pasternack BS \& Toniolo P 1997 Relation of serum levels of testosterone and dehydroepiandrosterone sulfate to risk of breast cancer in postmenopausal women. American Journal of Epidemiology 145 1030-1038. 